\title{
Three dimensional anatomy of the anterior cruciate ligament: a new approach in anatomical orthopedic studies and a literature review
}

This article was published in the following Dove Press journal:

Open Access Journal of Sports Medicine

9 November 2012

Number of times this article has been viewed

\author{
Gustavo Gonçalves Arliani' \\ Diego Costa Astur' \\ Eduardo Ramalho Moraes \\ Camila Cohen Kaleka ${ }^{2}$ \\ Wahi Jalikjian ${ }^{3}$ \\ Pau Golano ${ }^{4,5}$ \\ Moisés Cohen'
}

'Centro de Traumatologia do Esporte (CETE), Departamento de Ortopedia e Traumatologia da Universidade Federal de São Paulo SP, Brazil (DOTUNIFESP/EPM); ${ }^{2}$ Departamento de Ortopedia e Traumatologia Faculdade de Ciências Médicas da Santa Casa de São Paulo SP, Brazil; ${ }^{3}$ Departamento de Ortopedia e Traumatologia da Faculdade de Medicina de São José do Rio Preto SP, Brazil; ${ }^{4}$ Laboratory of Arthroscopic and Surgical Anatomy, Department of Pathology and Experimental Therapeutics, University of Barcelona, Spain; ${ }^{5}$ Department of Orthopedic Surgery, School of Medicine, University of Pittsburgh, Pittsburgh, PA, USA

Correspondence: Gustavo Arliani Rua Borges Lagoa, $783-5^{\circ}$ andar, Vila Clementino, São Paulo SP, Brazil 04038-032.

Tel +I I 55 557| 662 I

Email ggarliani@hotmail.com
Background: The anterior cruciate ligament (ACL) is an important structure in the knee. The ACL does not heal following lesions, and surgical reconstruction is the standard treatment among athletes. Some steps of ACL reconstruction remain controversial. It is important to fully understand the anatomy of the ACL to accurately reproduce its anatomy during surgical reconstructions. The purpose of this study was to evaluate the use of anaglyphic images that produce 3D images to better visualize the anatomy of the ACL, and to highlight the anatomical features of this ligament as reported in the literature.

Methods: We included ten knees in this study. After dissection of the knee structures, pictures were acquired using a camera with Nikon D40, AF-S Nikkor 18-55 mm (1:3.5-5.6 G2 ED), and Micro Nikkor $105 \mathrm{~mm}$ (1:2.8) lenses. The pair of images was processed using Callipyan 3D Anabuilder software, which transforms the two images into one anaglyphic image.

Results: During the dissection of the knees, nine pictures were acquired and transformed into anaglyphic images.

Conclusion: This study, demonstrated that the use of 3D images is a useful tool that can improve the knowledge of the anatomy of the knee, while also facilitating knee reconstruction surgery.

Keywords: anatomy education, photography methods, education, medical methods, eyeglasses, anaglyphic

\section{Introduction}

The anterior cruciate ligament (ACL) is an important structure in the knee, as it resists anterior instability and internal rotation of the tibia., ${ }^{1,2}$ The ACL does not heal following lesions, and surgical reconstruction is the standard treatment among athletes. ${ }^{3}$ Reconstructive surgery aims to restore the kinematics and stability of the injured knee. During the past 20 years, ACL reconstruction has become a widely accepted procedure, and its utilization will continue to increase given that the ACL is one of the most frequently injured structures during high impact sporting activities. ${ }^{4}$ However, some steps of ACL reconstruction remain controversial. It is important to fully understand the anatomy of the ACL in order to understand the functional role of this structure, as well as to accurately reproduce its anatomy during surgical reconstructions.

Several articles have been published on the anatomy of the ACL, mainly in relation to the footprints of this ligament in the femur and tibia. ${ }^{4-6}$ These studies aimed to increase the general knowledge of the anatomical features of the ACL in order to ensure that the present surgical techniques were as closely approximated as possible to 
the original anatomy of the ligament. However, there are no published studies on the anatomy of this ligament as viewed in three dimensions.

Two-dimensional images can be prepared to provide the illusion that an object has three dimensions (3D). Special glasses that transmit a different image of a two-dimensional object to each eye function by altering the angle of each image and produce an illusion of depth within the brain, or with the aid of computer graphics. Therefore, 3D visualization methods are a valuable resource for knee anatomy teaching and learning because they allow clinicians to visualize anatomical structures in great detail. The purpose of this study was to use anaglyphic images to visualize the anatomy of the ACL.

\section{Material and methods}

This research project was evaluated and approved by the Ethics Committee within our institution and an associated institution. We included ten knees from the traumatic amputation of lower limbs from adult patients between the ages of 34 and 55 years (mean 41.2 years). The knees of seven men and three women were dissected. There was no distinction based on race made among participants in the study. The dissections and 3D images were acquired at the Universidade Federal de São Paulo.

We did a qualitative analysis of the anatomy of the knee. The distal femur and proximal tibia were used for this study. The skin and subcutaneous tissue were removed, leaving only the muscle groups and ligaments. We then removed all of the muscle groups, and the ligaments and meniscus were retained. The quadriceps muscles, iliotibial band, biceps femoris, popliteus muscle, lateral and medial head of the gastrocnemius, semimembranosus, semitendinosus, gracilis, and sartorius were removed. Finally, we acquired a knee that only contained the bone (tibia and femur), the ACL, posterior cruciate ligament (PCL), medial collateral ligament, lateral collateral ligament, mucosum plica, and the meniscus. Following the dissection of each of these structures, pictures were taken with both central ligaments. Subsequently, the PCL was removed in order to better visualize the ACL.

In order to better visualize the two bundles of ACL in the flexion and extension positions, the medial condyle was resected with an osteotome. Images were then acquired with the knee in the flexion and extension positions. Images were acquired from the dissections using a camera with Nikon D40, AF-S Nikkor 18-55 mm (1:3.5-5.6 G2 ED), and Micro Nikkor 105 mm (1:2.8) lenses (Nikon Corporation, Tokyo, Japan). These lenses were placed on a slide bar that was attached to a tripod. The slider was used to generate two images of the same structure, which differed along the horizontal angle proportional to the interpupillary distance. ${ }^{7-9}$ The pair of images was processed using Callipyan 3D Anabuilder software (AnaBuilder, France), which transforms the two images into one anaglyphic image. The special anaglyph 3D glasses then enabled the visualization of the 3D images. Two images were displayed on the screen - one red and the other blue. The red filter allowed a red image to enter one eye, and the blue filter allowed a blue image to enter the other eye. The brain then combined these images to give the viewer the illusion of a 3D image.

\section{Results}

The distal femur and proximal tibia were used in this study. The skin, subcutaneous tissue and muscles were removed leaving only the femur, tibia, meniscus, and ligaments.

The first images were acquired just after a complete rupture of the patellar tendon and removal of the extensor mechanism proximally. In Figure 1 we can visualize the infrapatellar fat and the ligamentum mucosum. Following the acquisition of this image, we resected these two structures.

After dissection of ACL and PCL, we placed the joint at a 90-degree angle in flexion - a position typically used in arthroscopic procedures - to realize images in $3 \mathrm{D}$. In Figure 2, we can see both ligaments and their relations with the femur and tibia in the position described above.

With the knee in the same position, a new image (Figure 3 ) with a greater degree of zoom was acquired, so we were better able to observe the insertions of the ACL and PCL in 3D. Following that, the PCL was removed for better visualization of the ACL. Images were acquired from the front and back of the knee in different positions (Figures 4 and 5 , respectively). In the posterior view with the knee in the extended position, we can visualize more accurately the relationship between the ACL footprint and the posterior wall of the femur.

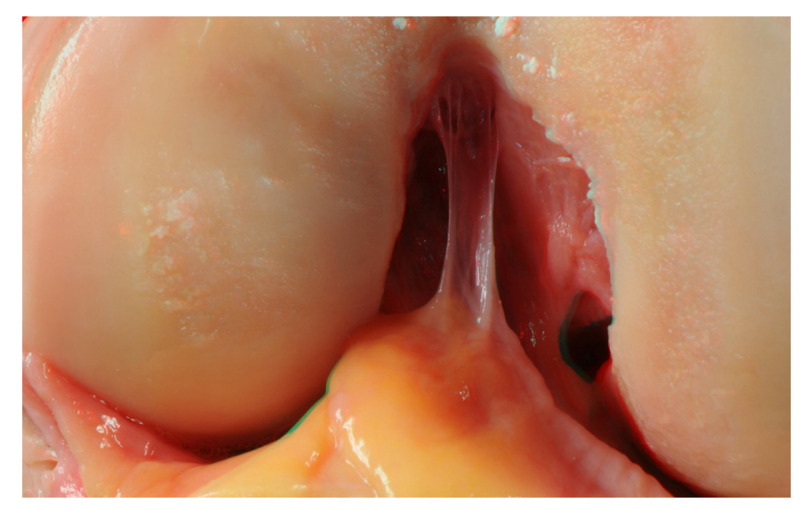

Figure I Infrapatellar fat and the ligamentum mucosum. 


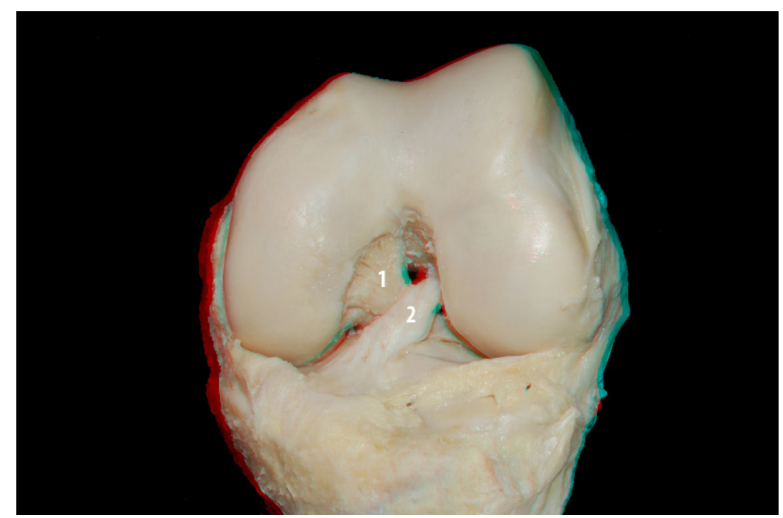

Figure $2 \mathrm{PCL}(\mathrm{I})$ and $\mathrm{ACL}(2)$ in knee flexion.

Abbreviations: $\mathrm{PCL}$, posterior cruciate ligament; $\mathrm{ACL}$, anterior cruciate ligament.

We then conducted a more detailed dissection of the ACL and its anteromedial and posterolateral bundles. To better visualize the two bundles in the flexion and extension positions, the medial condyle was resected with an osteotome. Images were then acquired with the knee in the flexed and extended positions (Figures 6 and 7, respectively). In Figure 8 it is apparent that when the knee is in flexion, the tension of the anteromedial bundle is clearly visible. It is also noted that the posterolateral bundle is loose. Also visible are the insertion of the femur and tibia.

With the knee in the extended position, and following resection of the medial femoral condyle, we performed a zoomed image at the femoral insertion of the ACL (Figure 9). In this image, we can see the footprint of the ligament as well as its relationship to the articular and posterior cortical region of the femur.

\section{Discussion}

The ACL is a band-like structure that is comprised of dense connective tissues. The ligament is composed of fibroblasts,

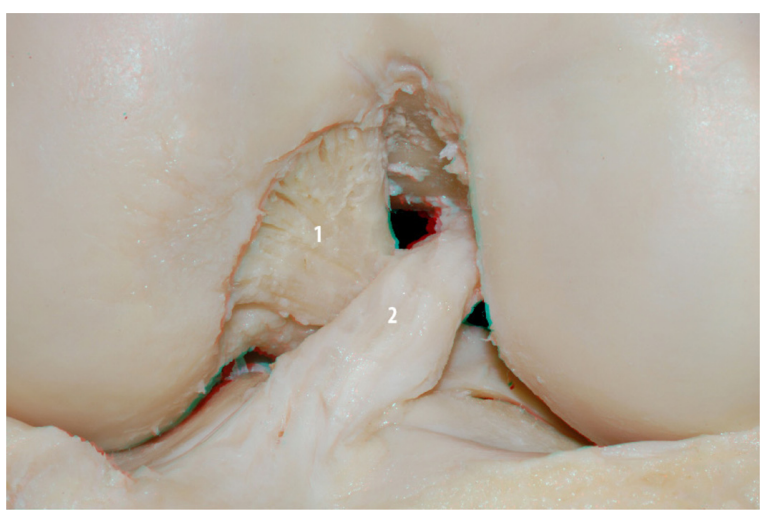

Figure $3 \mathrm{ACL}(2)$ and $\mathrm{PCL}(\mathrm{I})$ in the knee flexion.

Abbreviations: $\mathrm{PCL}$, posterior cruciate ligament; $\mathrm{ACL}$, anterior cruciate ligament.

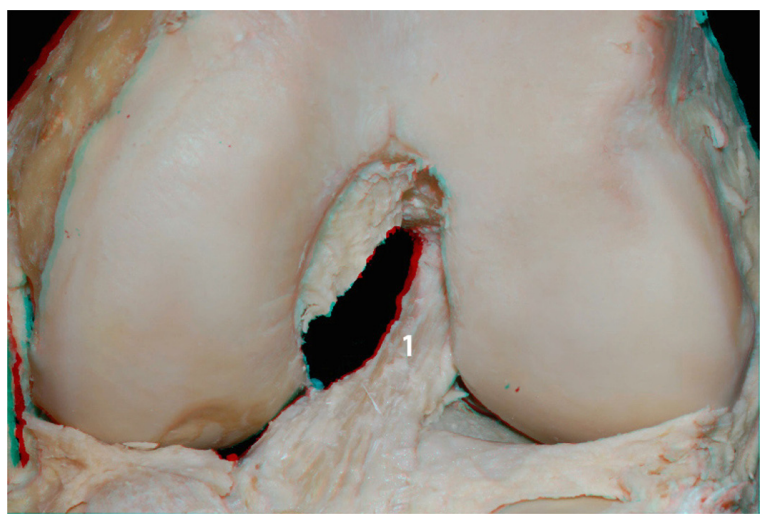

Figure $4 \mathrm{ACL}(\mathrm{I})$ in the flexed knee: anterior view.

Abbreviation: $A C L$, anterior cruciate ligament.

primarily surrounded by type 1 and type 3 collagens. Near the ACL insertions, there are also small amounts of type 3 and type 4 collagens. ${ }^{10,11}$

The normal insertion site of a ligament is a highly specialized zone of tissue that functions to transfer stress from hard to soft tissue. The native ACL attaches to the surface of the bone via direct insertion, which contains four distinct histological zones: (1) ligament; (2) uncalcified fibrocartilage; (3) calcified fibrocartilage; and (4) bone. ${ }^{12}$ The ACL attaches to a rough, nonarticular area, which is anterior to the intercondylar eminence of the tibia, and extends upwards and backwards in an oblique direction to the most posterior-medial aspect of the lateral femoral condyle. ${ }^{4}$ The long axis of the ACL is tilted 26 degrees forward from its vertical axis, and rotates towards itself in a lateral (external rotation) spiral of approximately 90 degrees as it approaches the tibial surface..$^{13,14}$

The ACL is enveloped by the synovial membrane of the knee joint, which places the ligament in an intraarticular orientation, although it remains in an extrasynovial position. ${ }^{15}$ The length of the ACL ranges from 22 to $41 \mathrm{~mm}$

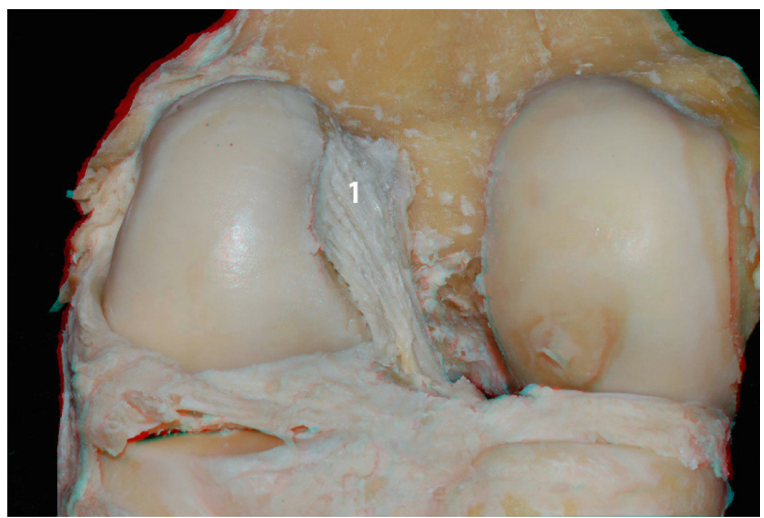

Figure 5 Femoral insertion of the $A C L(I)$ : posterior view. Abbreviation: $\mathrm{ACL}$, anterior cruciate ligament. 


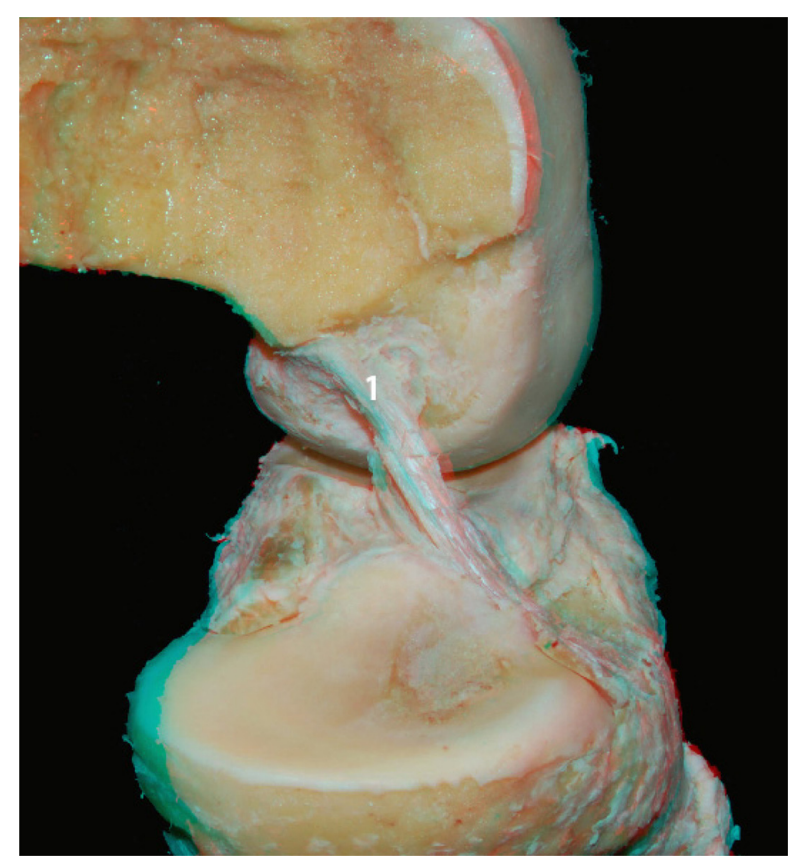

Figure $6 \mathrm{ACL}(\mathrm{I})$ without the medial condyle in the flexed knee: lateral view. Abbreviation: $A C L$, anterior cruciate ligament.

(mean, $32 \mathrm{~mm}$ ) and its width ranges from 7 to $12 \mathrm{~mm}$. The tibial attachment of the ACL is oval and longer in the anteroposterior dimension (17 $\pm 2 \mathrm{~mm}$ on average) than its medial-lateral width $\left(9 \pm 2 \mathrm{~mm}\right.$ on average). ${ }^{4}$ The femoral attachment is smaller and is located posterior and distal to the "over the top" position. The femoral attachment is oval shaped and there is often a bony prominence located between the anteromedial and posterolateral bundle insertion site, known as the cruciate ridge. In addition, there is a bony ridge running lengthwise throughout the entire footprint of the ACL, which is called the lateral intercondylar ridge. ${ }^{5}$

The cross-sectional shape of the ACL is noncircular. Moreover, the cross-sectional area increases in size from the

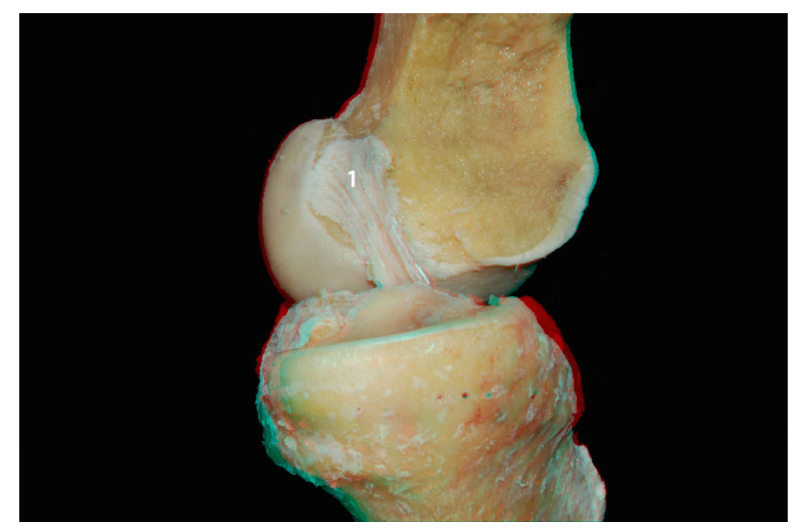

Figure $7 \mathrm{ACL}(\mathrm{I})$ without the medial condyle in the extended knee: lateral view. Abbreviation: $\mathrm{ACL}$, anterior cruciate ligament.

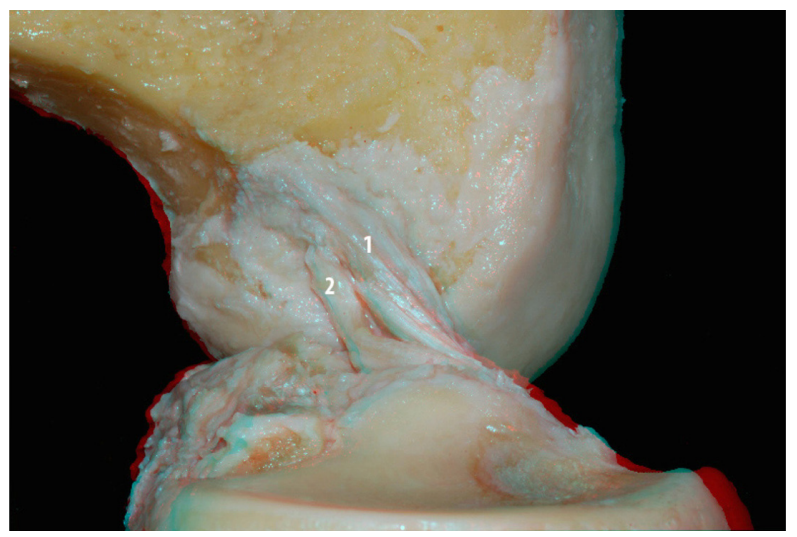

Figure $8 \mathrm{ACL}$ anteromedial (I) and posterolateral (2) bundles without the medial condyle: lateral view.

Abbreviation: $\mathrm{ACL}$, anterior cruciate ligament.

femur to the tibia. Near the tibial attachment, the ACL sends a variable amount of fibers anteriorly beneath the transverse intermeniscal ligament, and some extensions may blend in with both the attachment of the anterior or posterior horn of the lateral meniscus. The tibial attachment is somewhat wider and stronger than the femoral attachment. ${ }^{3}$

Functionally, Girgis et al divided the ACL into two parts: the anteromedial bundle (AMB) and the posterolateral bundle (PLB), ${ }^{14}$ while other orthopedic researchers have separated the ACL into three functional bundles: the AMB; the intermediate band; and the PLB. ${ }^{16}$ The fascicles of the AMB originate at the most anterior and proximal aspect of the femoral attachment and insert at the anteromedial aspect of the tibial attachment. Conversely, the fascicles of the PLB originate at the posterodistal aspect of the femoral attachment and insert at the posterolateral aspect of the tibial attachment. ${ }^{16}$ A larger number of fascicles make up the PLB as compared to the AMB. ${ }^{17}$ The two bundles are not isometric in terms of flexion and extension, but they

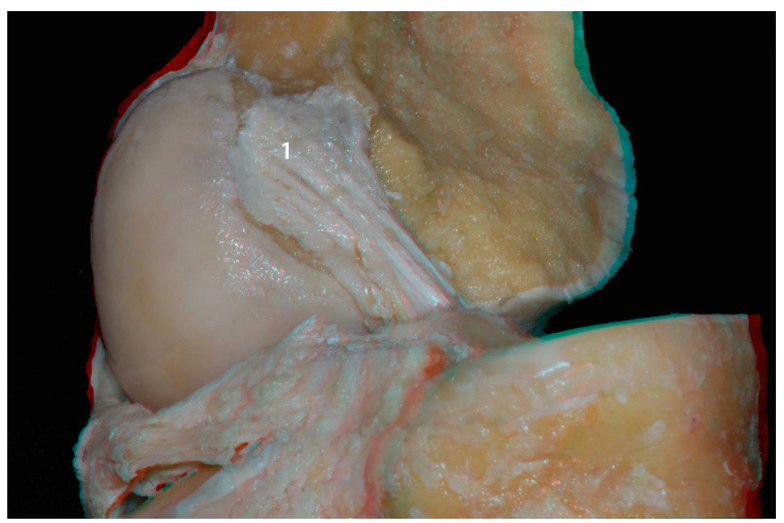

Figure $9 \mathrm{ACL}$ footprint (I) without the medial condyle. Abbreviation: $A C L$, anterior cruciate ligament. 
experience different patterns of changes in their length during passive knee flexion. In extension, the AMB appears as a flat band, and the posterolateral bulk of the ligament is taut. As the flexion begins, the anteromedial band tightens, and the posterolateral bulk loosens. ${ }^{4}$

In our study, we found two bundles in the ACL dissections, and as observed in other papers, we noted differences in the length of the ligament in different positions. The ACL receives nerve fibers from the posterior articular branches of the tibial nerve. These fibers penetrate the posterior joint capsule and run alongside the synovial and periligamentous vessels surrounding the ligament to reach as anterior as the infrapatellar fat pad. ${ }^{18}$ There are mechanoreceptors that serve a proprioceptive function and provide the afferent arc for knee postural signaling changes. ${ }^{3}$ Therefore, preserving ACL structural components during ACL reconstruction could help to maintain proprioception after reconstruction. ${ }^{19}$

The blood supply of the cruciate ligaments is provided by the middle genicular artery. The middle genicular artery originates at a right angle from the anterior aspect of the popliteal artery, most commonly at the level of the proximal contours of the femoral condyles immediately below the origin of the superior genicular artery and just above the sural artery. ${ }^{3}$ It pierces the posterior capsule, passing through one of the apertures existing in the oblique popliteal ligament, nearer to the lateral than the medial femoral condyle. When the artery penetrates the joint, it ramifies, providing branches to the soft tissues lodged in the intercondylar notch such as the ACL. ${ }^{3}$ The distribution of blood vessels within the ligament is not homogeneous. The proximal part of the ACL contains more blood vessels than the distal part. ${ }^{20}$

A small amount of blood is supplied to the distal portion of the ACL by the infrapatellar branches of the inferior genicular arteries. The periligamentous fold of vessels is absent in a small zone approximately $5-10 \mathrm{~mm}$ proximal to the tibial attachment; in this anterior fibrocartilaginous part, the tissue is avascular. ${ }^{21}$ The coincidence of poor vascularity and the presence of fibrocartilage is also observed in tendons that are subjected to compressive loads, and the coincidence of these two factors undoubtedly contributes to the poor healing potential of the ACL. ${ }^{3}$

\section{Conclusion}

The use of 3D images in this study demonstrates that this technique is useful to improve the knowledge of the anatomy of the knee, as well as to help inform knee reconstruction surgery. Other medical specialties have already used 3D imaging to enhance their understanding of various anatomical features. The ability to understand and visualize the relationship between different structures of the knee motivates us to conduct further studies using 3D images to improve our understanding of anatomical structures.

\section{Authors' contributions}

All authors substantially contributed to the conception and design of the study, acquisition of data, analysis and interpretation of data, drafting or reviewing the article and, if appropriate, revising it critically for important intellectual content. GGA, DCA, MC, ERM, and PG designed the study and directed its implementation, including the conduct of quality assurance, control, dissection, and acquisition of data. $\mathrm{CC}$ and WJ helped conduct the literature review and write the discussion section. All authors participated in all meetings during the preparation of the final document.

\section{Disclosure}

The authors report no conflict of interest in this work.

\section{References}

1. Matsumoto H, Suda Y, Otani T, Niki Y, Seedhom BB, Fujikawa K. Roles of the anterior cruciate ligament and the medial collateral ligament in preventing valgus instability. J Orthop Sci. 2001;6(1): 28-32.

2. Sakane M, Fox RJ, Woo SL, Livesay GA, Li G, Fu FH. In situ forces in the anterior cruciate ligament and its bundles in response to anterior tibial loads. J Orthop Res. 1997;15(2):285-293.

3. Duthon VB, Barea C, Abrassart S, Fasel JH, Fritschy D, Menetrey J. Anatomy of the anterior cruciate ligament. Knee Surg Sports Traumatol Arthrosc. 2006;14(3):204-213.

4. Giron F, Cuomo P, Aglietti P, Bull AM, Amis AA. Femoral attachment of the anterior cruciate ligament. Knee Surg Sports Traumatol Arthrosc. 2006;14(3):250-256.

5. Ferretti M, Ekdahl M, Shen W, Fu FH. Osseous landmarks of the femoral attachment of the anterior cruciate ligament: an anatomic study. Arthroscopy. 2007;23(11):1218-1225.

6. Tsukada H, Ishibashi Y, Tsuda E, Fukuda A, Toh S. Anatomical analysis of the anterior cruciate ligament femoral and tibial footprints. J Orthop Sci. 2008;13(2):122-129.

7. Ribas GC, Bento RF, Rodrigues AJ Jr. Anaglyphic three-dimensional stereoscopic printing: revival of an old method for anatomical and surgical teaching and reporting. J Neurosurg. 2001;95(6):1057-1066.

8. Shimizu S, Tanaka R, Rhoton AL Jr, et al. Anatomic dissection and classic three-dimensional documentation: a unit of education for neurosurgical anatomy revisited. Neurosurgery. 2006;58(5):E1000; discussion E1000.

9. Ribas GC, Ribas E Jr RA. The brain, the tridimensional vision, and the techniques to obtain stereoscopic images. Rev Med (Sao Paulo). 2006;85:78-90.

10. Baek GH, Carlin GJ, Vogrin TM, Woo SL, Harner CD. Quantitative analysis of collagen fibrils of human cruciate and meniscofemoral ligaments. Clin Orthop Relat Res. 1998;357:205-211.

11. Rumian AP, Wallace AL, Birch HL. Tendons and ligaments are anatomically distinct but overlap in molecular and morphological features - a comparative study in an ovine model. J Orthop Res. 2007;25(4):458-464.

12. Cooper RR, Misol S. Tendon and ligament insertion. A light and electron microscopic study. J Bone Joint Surg Am. 1970;52(1):1-20. 
13. Arnoczky SP. Anatomy of the anterior cruciate ligament. Clin Orthop Relat Res. 1983;172:19-25.

14. Girgis FG, Marshall JL, Monajem A. The cruciate ligaments of the knee joint. Anatomical, functional and experimental analysis. Clin Orthop Relat Res. 1975;106:216-231.

15. Ellison AE, Berg EE. Embryology, anatomy, and function of the anterior cruciate ligament. Orthop Clin North Am. 1985;16(1):3-14.

16. Amis AA, Dawkins GP. Functional anatomy of the anterior cruciate ligament. Fibre bundle actions related to ligament replacements and injuries. J Bone Joint Surg Br. 1991;73(2):260-267.

17. Souryal TO, Moore HA, Evans JP. Bilaterality in anterior cruciate ligament injuries: associated intercondylar notch stenosis. Am J Sports Med. 1988;16(5):449-454.
18. Kennedy JC, Alexander IJ, Hayes KC. Nerve supply of the human knee and its functional importance. Am J Sports Med. 1982;10(6): 329-335.

19. Adachi N, Ochi M, Uchio Y, Iwasa J, Ryoke K, Kuriwaka M. Mechanoreceptors in the anterior cruciate ligament contribute to the joint position sense. Acta Orthop Scand. 2002;73(3):330-334.

20. Scapinelli R. Vascular anatomy of the human cruciate ligaments and surrounding structures. Clin Anat. 1997;10(3):151-162.

21. Petersen W, Tillmann B. Structure and vascularization of the cruciate ligaments of the human knee joint. Anat Embryol (Berl). 1999;200(3): $325-334$.

\section{Publish your work in this journal}

Open Access Journal of Sports Medicine is an international, peer-reviewed, open access journal publishing original research, reports, reviews and commentaries on all areas of sports medicine. The manuscript management system is completely online and includes a very quick and fair peer-review system.
Visit http://www.dovepress.com/testimonials.php to read real quotes from published authors. 